\title{
Editorial Note: Frontiers in Multimedia Analytics Emerging Media Types Technologies and Applications
}

Published online: 8 April 2019

(C) Springer Science+Business Media, LLC, part of Springer Nature 2019

Multimedia Tools and Applications gratefully acknowledges the editorial work of the scholars listed below on the special issue entitled, "Frontiers in Multimedia Analytics Emerging Media types Technologies and Applications."

Of 29 papers submitted to this issue, 14 were eventually accepted after a stringent peer-review process.

Dr. Jitao Sang (Corresponding Guest Editor)

Chinese Academy of Sciences, Beijing, China

jtsang@nlpr.ia.ac.cn

\section{Dr. Zechao Li}

Nanjing University of Science and Technology, Nanjing, China zechao.li@njust.edu.cn

\section{Dr. Zhineng Chen}

Chinese Academy of Sciences, Beijing, China

zhineng.chen@ia.ac.cn

\section{Prof. Abdulmotaleb El Saddik}

University of Ottawa, Ottawa, Canada

elsaddik@uottawa.ca

Publisher's Note Springer Nature remains neutral with regard to jurisdictional claims in published maps and institutional affiliations. 